\title{
Influence of neighbourhood-level crowding on sleep-disordered breathing severity: mediation by body size
}

\author{
DAYNA A. JOHNSON ${ }^{1,2,3}$, CHRISTOPHER DRAKE ${ }^{4}$, CHRISTINE \\ L. M. JOSEPH ${ }^{3}$, RICHARD KRAJENTA ${ }^{3}$, DAVID W. HUDGEL ${ }^{5}$ and ANDREA \\ E. CASSIDY-BUSHROW ${ }^{3}$ \\ ${ }^{1}$ Department of Epidemiology, University of Michigan, Ann Arbor, MI, USA; ${ }^{2}$ Division of Sleep and Circadian Disorders, Brigham and Women's \\ Hospital and Harvard Medical School, Boston, MA, USA; ${ }^{3}$ Department of Public Health Sciences and the Health Disparities Research \\ Collaborative, Henry Ford Hospital, Detroit, MI, USA; ${ }^{4}$ Sleep Disorders and Research Center, Henry Ford Hospital, Detroit, MI, USA; and \\ ${ }^{5}$ University of Manitoba, 373 Misericordia Health Centre, Winnipeg, MB, Canada
}

\author{
Keywords \\ apnoa-hypopnoea index, body mass index, \\ mediation analysis, sleep, social determinants of \\ sleep, polysomnography

\section{Correspondence} \\ Andrea E. Cassidy-Bushrow, PhD, MPH, One \\ Ford Place Suite $5 \mathrm{C}$, Henry Ford Health \\ System, Detroit, MI 48202, USA. \\ Tel.: +313 8746097 ; \\ fax: +313 8746656 ; \\ e-mail: acassid1@hfhs.org
}

Accepted in revised form 4 April 2015; received 12 January 2015

DOI: $10.1111 /$ jsr.12305

\section{SUMMARY}

Neighbourhood-level crowding, a measure of the percentage of households with more than one person per room, may impact the severity of sleep-disordered breathing. This study examined the association of neighbourhood-level crowding with apnoea-hypopnoea index in a large clinical sample of diverse adults with sleep-disordered breathing. Sleepdisordered breathing severity was quantified as the apnoea-hypopnoea index calculated from overnight polysomnogram; analyses were restricted to those with apnoea-hypopnoea index $\geq 5$. Neighbourhoodlevel crowding was defined using 2000 US Census tract data as percentage of households in a census tract with $>1$ person per room. Multivariable linear mixed models were fit to examine the associations between the percentage of neighbourhood-level crowding and apnoeahypopnoea index, and a causal mediation analysis was conducted to determine if body mass index acted as a mediator between neighbourhood-level crowding and apnoea-hypopnoea index. Among 1789 patients (43\% African American; 68\% male; $80 \%$ obese), the mean apnoea-hypopnoea index was $29.0 \pm 25$.3. After adjusting for race, age, marital status and gender, neighbourhood-level crowding was associated with apnoea-hypopnoea index; for every one-unit increase in percentage of neighbourhood-level crowding mean, the apnoea-hypopnoea index increased by $0.40 \pm 0.20(P=0.04)$. There was a statistically significant indirect effect of neighbourhood-level crowding through body mass index on the apnoea-hypopnoea index $(P<0.001)$. Neighbourhood-level crowding is associated with severity of sleep-disordered breathing. Body mass index partially mediated the association between neighbourhoodlevel crowding and sleep-disordered breathing. Investigating prevalent neighbourhood conditions impacting breathing in urban settings may be promising.

\section{INTRODUCTION}

Little is known about the social determinants of disordered sleep (Patel et al., 2010). Growing research suggests neighbourhood environment is associated with sleep characteristics and adherence to sleep therapy (Desantis et al., 2013; Grandner et al., 2013; Hale and Do, 2007; Hale et al., 2013; Platt et al., 2009). Prior studies have shown that residing in a disadvantaged neighbourhood is associated with poor sleep duration, sleep quality and sleep-disordered breathing (SDB; Brouillette et al., 2011; Desantis et al., 2013; Hale and Do, 2007; Spilsbury et al., 2006). These studies are typically limited in sample size and diversity of the study sample. Disparities in sleep could be partially attributed to differences in the neighbourhood environment. Differentiating dimensions of the neighbourhood environment is necessary in order to determine features in the neighbourhood that may contribute to poor sleep outcomes. 
Household crowding is most commonly defined as a housing unit with more than one person per room in the residence (Blake et al., 2007), and can be defined at the neighbourhood-level using census data. Household crowding is associated with poor health, including psychological stress, mental health outcomes and obesity (Cardoso et al., 2004; Evans et al., 1989; Leventhal and Brooks-Gunn, 2003). Crowding is most prominent among minority populations, and in urban areas with a higher percentage of renters and lower-income households (Blake et al., 2007). There are a few hypothesized mechanisms that may explain the association of crowding and poorer health measures, including SDB. First, crowding may negatively impact activity level or lead to an increased perceived stress level (Fuller et al., 1993; Rohe and Han, 2012). Second, a limited amount of space per person may promote the spread of communicable diseases, leading to establishment or exacerbation of diseases such as bronchitis, pneumonia and asthma (Fuller et al., 1993; Krieger and Higgins, 2002; Rohe and Han, 2012). Several studies have demonstrated an association of crowding with respiratory infections among children and adults (Denny, 1995; Fonseca et al., 1996; Graham, 1990; Krieger and Higgins, 2002; Murtagh et al., 1993; Rohe and Han, 2012). In youth, respiratory concerns including sinus problems and persistent wheeze independently predict SDB measured as the apnoea-hypopnoea index $(\mathrm{AHI}) \geq 10$ (Redline et al., 1999). Crowding is also associated with obesity. Data from the Coronary Artery Risk Development in Young Adults (CARDIA) study, a large cohort of blacks and whites in the USA, showed that housing density (defined as the ratio of people to bedrooms in the home) increased the risk of obesity (Chambers et al., 2010), an established risk factor for SDB (Baldwin and Quan, 2002). Thus, by promoting respiratory problems and/or increasing the risk of obesity, overcrowded housing conditions could lead to increased severity of SDB.

To our knowledge, however, no study has examined the association of neighbourhood-level crowding with SDB severity measured with $\mathrm{AHI}$, in a sample of adults. We examined the association of neighbourhood-level crowding with $\mathrm{AHI}$ in a large sample of diverse adults with SDB from a single health system, overall and by race and by gender. Secondly, we examined if body mass index (BMI) mediated the overall association of neighbourhood-level crowding with $\mathrm{AHI}$.

\section{MATERIALS AND METHODS}

Between 2001 and 2008, a total of 4905 clinical patients were seen for a sleep study at Henry Ford Health System (HFHS; Detroit, MI, USA), and were part of the clinical registry. All patients had an overnight polysomnography (PSG) in the HFHS sleep clinic as part of clinical care. The PSG was used to calculate the $\mathrm{AHI}$, defined as the number of apnoeas or hypopnoeas per hour of sleep. Race was obtained from the electronic medical record (EMR). Height and weight closest to but before the time of the sleep study clinic visit (within 2 years) were obtained from the EMR and used to calculate BMI $\left(\mathrm{kg} \cdot \mathrm{m}^{-2}\right)$.

There was a total of 3761 patients with probable SDB based on the $\mathrm{AHI} \geq 5$ (as defined in a similar study; Pranathiageswaran et al., 2013). Seventy-nine patients with missing data, 82 children ( $<18$ years old), 410 of non-African American or Caucasian race, and 418 living outside metropolitan Detroit area or who had addresses not mappable to the US census were excluded from the analysis. An additional 983 patients for whom BMl was not obtainable from the EMR were also excluded. The final analytic sample consisted of 1789 adults. The study was approved by the institutional review board at HFHS.

\section{Geocoding}

Patient addresses at the time of PSG were mapped to the 2000 US Census Bureau census tract using a commercial package assembled by Mapping Solutions, LLC Lansing, MI, USA. The components include MapInfo Professional and MapMarker, Maplnfo Corporation Troy, NY, USA. The geocoded addresses were used to determine the percentage of neighbourhood-level crowding at the census tract level. Neighbourhood-level crowding was defined as the percentage of households within a census tract with more than one person per room, a standard measure of overcrowding (Gove et al., 1979).

\section{Analyses}

A statistical significance level of 0.05 was used. All tests were two-sided. For descriptive purposes, characteristics were compared by race using a chi-square test for discrete variables and a $t$-test for continuous variables.

We first sought to examine if there was an overall association between neighbourhood-level crowding and SDB severity, measured as the continuous AHI. Linear mixed models were used to account for potential correlation between patients residing in the same census tract. Models were fit: unadjusted (Model I); and adjusted for age, marital status, race and gender (Model II). Race and gender have recently been shown to be associated with SDB severity (Pranathiageswaran et al., 2013). As the impact of social determinants on health can vary by race and gender, we refit the models described above also stratified by race and by gender (Ompad et al., 2007).

In the full sample, we utilized the Baron and Kenny (1986) four-step approach to examine if BMl acted as a mediator variable in the relationship between neighbourhood-level crowding and AHI (Baron and Kenny, 1986). First, we examined the association between neighbourhood-level crowding and AHI. Second, we examined if neighbourhoodlevel crowding was significantly associated with BMI. Third, we examined if BMI was associated with AHI. Statistically significant associations need to be detected in steps 1-3 to 
continue evaluating for mediation. Finally, we fit a model of $\mathrm{AHI}$ with both neighbourhood-level crowding and $\mathrm{BMI}$ in the model (Model III). When the $\beta$ in the model adjusted for the potential mediator (in this case, $\mathrm{BMI}$ ) is smaller than the $\beta$ in the model without the potential mediator, this indicates partial mediation. If the $\beta$ in the model adjusted for the potential mediator (here BMI) approaches 0 , that indicates complete mediation. The causal mediation analysis approach (Imai et al., 2010a,b; Tingley et al., 2013) was used to estimate the average causal mediation effect (ACME), the average direct effect and the proportion mediated.

We also conducted several sensitivity analyses. First, we examine quartiles of neighbourhood-level crowding with $\mathrm{AHI}$ because continuous neighbourhood variables sometimes overestimate effects (Mooney et al., 2014). Second, associations between neighbourhood crowding, BMI and SDB severity could be confounded by other neighbourhood socioeconomic status (SES) indicators. We examined if adjusting our models for neighbourhood-level poverty impacted our findings.

\section{RESULTS}

For descriptive purposes, study population characteristics are presented by race in Table 1. Among our clinic-based sample, the overall mean $\mathrm{AHI}$ was $29.0 \pm 25.3$; $\mathrm{AHI}$ did not differ by race $(P=0.70)$. However, obstructive apnoeas were higher among African Americans, whereas obstructive and central hypopnoeas were higher among non-Hispanic whites. Non-Hispanic white patients were more likely to be older, married, male, have a higher income, employed, to be living in a neighbourhood with a greater number of households

\begin{tabular}{|c|c|c|c|}
\hline & $\begin{array}{l}\text { African } \\
\text { American }\end{array}$ & $\begin{array}{l}\text { Non-Hispanic } \\
\text { white }\end{array}$ & $P$ \\
\hline$N$ & 768 & 1021 & - \\
\hline Age (years) & $52.1 \pm 13.1$ & $56.5 \pm 12.3$ & $<0.001$ \\
\hline BMI $\left(\mathrm{kg} \mathrm{m}^{-2}\right)$ & $40.0 \pm 9.1$ & $35.9 \pm 8.7$ & $<0.001$ \\
\hline Married & $366(20.5 \%)$ & $723(40.4 \%)$ & $<0.001$ \\
\hline Male & 464 (25.9\%) & 747 (41.8\%) & $<0.001$ \\
\hline $\begin{array}{l}\text { Neighbourhood } \\
\text { crowding (\%) }\end{array}$ & $5.6 \pm 3.5$ & $2.6 \pm 2.9$ & $<0.001$ \\
\hline $\begin{array}{l}\text { Neighbourhood } \\
\text { poverty level (\%) }\end{array}$ & $18.6 \pm 11.8$ & $6.3 \pm 7.0$ & $<0.001$ \\
\hline $\begin{array}{l}\text { Obstructive } \\
\text { apnoeas }\end{array}$ & $17.0 \pm 23.7$ & $13.3 \pm 19.3$ & $<0.001$ \\
\hline $\begin{array}{l}\text { Obstructive } \\
\text { hypopnoeas }\end{array}$ & $6.3 \pm 9.5$ & $7.4 \pm 10.0$ & 0.02 \\
\hline Central apnoeas & $2.2 \pm 7.5$ & $2.6 \pm 7.2$ & 0.20 \\
\hline $\begin{array}{l}\text { Central } \\
\text { hypopnoeas }\end{array}$ & $1.8 \pm 3.9$ & $2.6 \pm 5.1$ & $<0.001$ \\
\hline $\mathrm{AHI}$ & $29.2 \pm 26.6$ & $28.8 \pm 24.2$ & 0.70 \\
\hline
\end{tabular}

below poverty level, and to have a smaller BMI than African American patients (all $P<0.05$ ).

In our sample, participants lived in neighbourhoods where the mean level of crowding was $3.9 \pm 3.5 \%$. This estimate was statistically significantly higher among African American patients (5.6\%) than non-Hispanic white patients $(2.6 \% ; P<$ 0.001 ; Table 1). Additionally, BMI and neighbourhood-level crowding were significantly and positively associated ( $\beta=$ 0.58, $P<0.001)$. In contrast, age, male gender and marital status were negatively associated with neighbourhood-level crowding $(\beta=-0.46, P<0.001 ; \beta=-0.05, P<0.001$; and $\beta=-0.11, P<0.001$, respectively).

\section{Association of neighbourhood-level crowding with $\mathrm{AHI}$}

Table 2 describes the association of neighbourhood-level crowding with $\mathrm{AHI}$ in the full sample. In the unadjusted analysis, neighbourhood-level crowding was associated with $\mathrm{AHI}$; for every one-unit change in neighbourhood-level crowding there was a corresponding predicted average change in $\mathrm{AHI}$ of $0.37 \pm 0.17(P=0.03$; Model I). After adjusting for race, age, marital status and gender, the association between neighbourhood-level crowding and $\mathrm{AHI}$ persisted ( $\beta=0.40, P=0.04$; Model II).

We fit additional models to examine the association of neighbourhood-level crowding and $\mathrm{AHI}$ stratified by gender or race (Table 3 ). In gender-specific models, there was a positive association between neighbourhood-level crowding and $\mathrm{AHI}$ in men $(\beta=0.55, P=0.01)$, but not in women $(\beta=0.33, P=0.19)$. The association in men was attenuated after adjusting for covariates $(\beta=0.21, P=0.38)$. In racespecific models, neighbourhood-level crowding was positively associated with $\mathrm{AHI}$ among African Americans $(\beta=0.63$, $P=0.02)$. This association remained robust to covariate adjustment $(\beta=0.59, P=0.03)$, and was attenuated but remained marginally statistically significant in the fullyadjusted model that included $\mathrm{BMI}(\beta=0.48, P=0.07)$. Male gender and BMI had a positive significant effect in the stratified models for African Americans. Among non-Hispanic whites, there was no statistically significant association between neighbourhood-level crowding and $\mathrm{AHI}(\beta=0.16, P=0.54)$. Male gender and BMI were positively and marital status (Model 2) was negatively associated with $\mathrm{AHI}$ in the non-Hispanic white-only model.

\section{BMI mediates the association between neighbourhood- level crowding and $\mathrm{AHI}$}

The mediation analysis in the full sample is presented in Fig. 1. As described above, neighbourhood-level crowding was significantly associated with $\mathrm{AHI}(\beta=0.37, P=0.03)$ and with BMI ( $\beta=0.58, P=0.01$; Fig. 1 ; Table 2$)$. BMI was also statistically significantly associated with $\mathrm{AHI} \quad(\beta=0.45$, $P<0.01$; Fig. 1). We further compared the coefficients for neighbourhood-level crowding found in Model I $(\beta=0.37)$ and Model III $(\beta=0.20)$, demonstrating a reduction once BMI was 
Table 2 Association of neighbourhood crowding with $\mathrm{AHI}$, in the full sample $(N=1789)$

\begin{tabular}{|c|c|c|c|}
\hline Covariate & $\begin{array}{l}\text { Model I } \beta \pm S E \\
(P \text {-value })\end{array}$ & $\begin{array}{l}\text { Model II } \beta \pm S E \\
(\mathrm{P} \text {-value) }\end{array}$ & $\begin{array}{l}\text { Model III } \beta \pm S E \\
(\mathrm{P} \text {-value) }\end{array}$ \\
\hline Neighbourhood-level crowding & $\begin{array}{l}0.37 \pm 0.17 \\
(P=0.03)\end{array}$ & $\begin{array}{l}0.40 \pm 0.2 \\
(P=0.04)\end{array}$ & $\begin{array}{l}0.20 \pm 0.2 \\
(P=0.29)\end{array}$ \\
\hline African American & & $\begin{array}{l}-0.50 \pm 1.3 \\
(P=0.71)\end{array}$ & $\begin{array}{l}-1.4 \pm 1.3 \\
(P=0.27)\end{array}$ \\
\hline Age (years) & & $\begin{array}{l}-0.10 \pm 0.05 \\
(P=0.04)\end{array}$ & $\begin{array}{l}-0.02 \pm 0.05 \\
(P=0.72)\end{array}$ \\
\hline Married & & $\begin{array}{l}-2.2 \pm 1.3 \\
(P=0.08)\end{array}$ & $\begin{array}{l}-1.8 \pm 1.3 \\
(P=0.15)\end{array}$ \\
\hline Male & & $\begin{array}{l}8.9 \pm 1.3 \\
(P<0.001)\end{array}$ & $\begin{array}{l}11.1 \pm 1.3 \\
(P<0.001)\end{array}$ \\
\hline $\mathrm{BMI}\left(\mathrm{kg} \cdot \mathrm{m}^{-2}\right)$ & & & $\begin{array}{l}0.56 \pm 0.1 \\
(P<0.001)\end{array}$ \\
\hline
\end{tabular}

$\beta$, parameter estimate; BMI, body mass index; SE, standard error.

Model I: unadjusted; Model II: adjusted for race, age, marital status and gender; Model III: additionally adjusted for BMI.

Table 3 Gender and race-specific association of neighbourhood crowding with $\mathrm{AHI}$

\begin{tabular}{llll}
\hline $\begin{array}{l}\text { Neighbourhood } \\
\text { crowding }\end{array}$ & $\begin{array}{l}\text { Model I } \\
\beta \pm S E \\
(P \text {-value) }\end{array}$ & $\begin{array}{l}\text { Model II } \\
\beta \pm S E \\
\text { (P-value) }\end{array}$ & $\begin{array}{l}\text { Model III } \\
\beta \pm S E \\
(P \text {-value) }\end{array}$ \\
\hline $\begin{array}{l}\text { Gender-specific } \\
\text { models }\end{array}$ & & & \\
Male & $0.55 \pm 0.2$ & $0.40 \pm 0.2$ & $0.21 \pm 0.2$ \\
Female & $(P=0.01)$ & $(P=0.11)$ & $(P=0.38)$ \\
Race-specific & $0.33 \pm 0.2$ & $0.38 \pm 0.3$ & $0.20 \pm 0.3$ \\
models & $(P=0.19)$ & $(P=0.17)$ & $(P=0.46)$ \\
African & & & \\
$\quad \begin{array}{l}\text { American } \\
\text { Non-Hispanic }\end{array}$ & $0.63 \pm 0.3$ & $0.59 \pm 0.3$ & $0.48 \pm 0.3$ \\
white & $(P=0.02)$ & $\begin{array}{l}(P=0.03) \\
(P=0.07)\end{array}$ \\
\hline
\end{tabular}

$\beta$, parameter estimate; SE, standard error.

Model I: unadjusted; Model II: adjusted for race, age, marital status and gender; Model III: additionally adjusted for BMI.

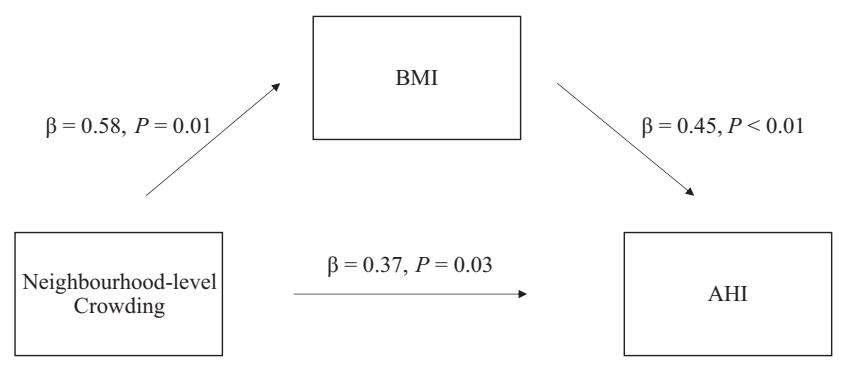

Figure 1. Mediation analysis presenting evidence that body mass index (BMI) may mediate the association between neighbourhood-level crowding and sleep-disordered breathing (SDB) severity measured as apnoea-hypopnoea index (AHI). $\beta$, parameter estimate. The indirect effect was also calculated $(\beta=$ $0.26, P<0.01)$. added to the model. There was a statistically significant ACME $(P<0.001)$ suggesting that there is a significant indirect effect of neighbourhood crowding, through BMI, on $\mathrm{AHI}$; the proportion of the association of neighbourhood-level crowding on $\mathrm{AHI}$ mediated by $\mathrm{BMI}$ was $71.8 \%(P=0.08)$. In the causal mediation analysis model, there was no evidence for a direct effect between neighbourhood-level crowding and AHI $(P=0.58)$.

\section{Association of neighbourhood-level crowding with other respiratory sleep variables}

We fit additional models to explore the associations of neighbourhood-level crowding with other respiratory sleep variables. Neighbourhood-level crowding was associated with obstructive apnoea ( $\beta=0.54, P<0.001)$; after covariate adjustment, however, this was attenuated and no longer statistically significant $(\beta=0.21, P=0.18)$. Neighbourhoodlevel crowding was not associated with any other respiratory sleep variable available (all $P>0.10$ ).

\section{Sensitivity analysis}

We examined quartiles of neighbourhood-level crowding with AHI. Those in the 4th compared with 1st quartile of neighbourhood-level crowding had a higher AHI before adjustment for $\mathrm{BMI}$ (overall variable $P=0.02$ ), but the association was attenuated after adjustment for BMI. Results were also similar after additionally adjusting for neighbourhood-level poverty.

\section{DISCUSSION}

We provide new evidence suggesting that neighbourhoodlevel crowding is associated with severity of SDB $(A H I \geq 5)$ among a clinic-based population. This association appears to be partially mediated by BMI. Additionally, the association 
between neighbourhood-level crowding and SDB severity was stronger among African American patients; however, the association was marginally significant in fully adjusted models.

Neighbourhood-level crowding could be a proxy of a poor housing environment; for example, living in poor housing conditions can increase exposure to allergens and subsequently increase AHI (Spilsbury et al., 2006), and the impact of these exposures on $\mathrm{AHI}$ might vary by airway anatomy. Neighbourhood-level crowding may be one contributor among others that may contribute to SDB severity. The association between neighbourhood-level crowding with SDB severity appeared to be driven primarily by the obstructive apnoeas, which is consistent with these potential mechanisms. Although more research is needed, neighbourhoodlevel crowding may serve as an indicator for identifying patients that are at higher risk for more severe SDB as measured by the $\mathrm{AHI}$.

Obesity is one of the strongest known risk factors for SDB presence (Peppard et al., 2013) and severity (Peppard et al., 2000), and is also associated with crowding (Chambers et al., 2010). Our results suggest that the overall association between neighbourhood-level crowding and SDB severity is mediated through BMI. There are several ways BMI may mediate the neighbourhood-level crowding and $\mathrm{AHI}$ association. Household crowding is related to limited physical activity and increased distress (Fuller et al., 1993; Rohe and Han, 2012). Physical activity level may be diminished in crowded homes, thus leading to increased body size and therefore increased SDB severity. Perceived and biologically measured stress is associated with obesity, particularly upper body obesity; upper body obesity is similarly associated with sleep apnoea, thus providing another potential mechanism by which BMI may mediate the neighbourhood-level crowding and AHI relation (Bose et al., 2009; Millman et al., 1995; Young et al., 1993).

In contrast to Pranathiageswaran et al. (2013), we did not find an overall difference in SDB severity comparing African American with non-Hispanic white patients in our sample. Our sample was older and more predominately male than this previous study, which could explain the different findings. In our study, however, we did find evidence for a race-specific effect of neighbourhood-level crowding on AHI. In our racespecific models, neighbourhood-level crowding was positively associated with $\mathrm{AHI}$ among African Americans only, and this association persisted, although it was slightly attenuated, even after adjustment for BMI. Pranathiageswaran et al. (2013) hypothesize that anatomic differences, including upper airway mechanics, in African Americans compared with non-Hispanic whites could be related to racial differences in SDB severity. Researchers have suggested that the more severe SDB among African Americans than other races found in other studies may be a result from a delay in seeking treatment (Ruiter et al., 2010). This could be the result of other relevant factors such as limited access to resources or care, SES, educational status, or other deter- minants of racial disparities (Pranathiageswaran et al., 2013), all of which may also be related to neighbourhood crowding. Even after adjusting for neighbourhood-level poverty in our race-specific models, neighbourhood-level crowding remained associated with $\mathrm{AHI}$ in our African American patients, suggesting that neighbourhood SES may not be accounting for the race-specific association of neighbourhood-level crowding on $\mathrm{AHI}$.

The association between neighbourhood-level crowding and SDB severity was statistically significant; however, the magnitude of the association was relatively small. If we instead interpret the parameter estimate for a 1-unit standard deviation change in neighbourhood-level crowding (3.5\%), we would expect an increase in $\mathrm{AHI}$ of $\sim 1.4$ events per hour. Even relatively small changes in $\mathrm{AHI}$ are associated with relatively large increases in medical costs, particularly in the mild-tomoderate SDB range (Kapur et al., 1999), and a change of only 2.8 events per hour is associated with incident cardiovascular disease in patients with SDB (Chami et al., 2011).

There are several strengths to our study. First, we utilized an objective measure of SDB, AHI by overnight PSG. Second, our patient population was racially diverse. Lastly, we documented an association between the neighbourhood environment and sleep in a large adult population.

There are limitations to our study. As done by others, we restricted our analysis of SDB severity to those with an $\mathrm{AHI} \geq$ 5 (Pranathiageswaran et al., 2013) in order to identify a population with SDB; however, other lower bounds of disease may be more appropriate. However, we examined SDB as a continuous variable in sensitivity analyses including the entire population $(\mathrm{AHI} \geq 0$ ), and the results were consistent with that of $\mathrm{AHI} \geq 5$. An additional limitation is that there were no measures of individual SES available. Not all participants had an available BMI measure at the same time as the PSG; however, nearly half had a BMI measure within 1 month of the PSG, and the mean time between BMI and PSG measurement was only $6.0 \pm 6.1$ months. In a longitudinal study of older adults, there were only minimal changes in BMI over each 2year increment of measure (Botoseneanu and Liang, 2012). In a study of weight change and sleep apnoea, among participants who at baseline did not have sleep apnoea but developed it over a 4-year follow-up period, the mean weight change was only $1.0 \mathrm{~kg}$ year $^{-1}$ (Peppard et al., 2000); thus, it is unlikely there were significant changes in BMI during our relatively short study duration time-frame. This is a clinic-based sample of referred patients, which may not be representative of the general population. In order to conduct our mediation analysis, we needed to make assumptions regarding temporality of our measures (i.e. that BMI preceded SDB), which may be incorrect. Given the cross-sectional design of our study, we are unable to assess causality.

In summary, we found a novel association between neighbourhood-level crowding and $\mathrm{AHI}$, and there was evidence of partial mediation of this association by BMI. The association was more pronounced among African Americans in our study sample, and suggests that 
neighbourhood conditions may contribute to potential race-disparities in healthy sleep. Other aspects of the neighbourhood and $\mathrm{AHI}$ should be further examined in samples of diverse adults. Investigating prevalent neighbourhood conditions that directly affect breathing such as pollution or cockroaches in urban settings may be promising. Identifying health-deteriorating aspects of the neighbourhood could assist in targeting interventions to improve neighbourhood conditions and consequently health.

\section{AUTHOR CONTRIBUTION}

Statistical analysis and drafting of the manuscript: DAJ, AECB; critical revision of the manuscript: DAJ, CD, CLMJ, RK, DWH, AECB; geocoding: RK; study supervision: AECB.

\section{CONFLICT OF INTEREST}

All authors have indicated no financial conflicts of interest.

\section{REFERENCES}

Baldwin, C. M. and Quan, S. F. Sleep disordered breathing. Nurs. Clin. North Am., 2002, 37: 633-654, vi.

Baron, R. M. and Kenny, D. A. The moderator-mediator variable distinction in social psychological research: conceptual, strategic, and statistical considerations. J. Pers. Soc. Psychol., 1986, 51: 1173-1182.

Blake, K. S., Kellerson, R. L. and Simic, A. Measuring Overcrowding in Housing. U.S. Department of Housing and Urban Development. Office of Policy Develpment and Research, Available online at: http://www.huduser.org/publications/pdf/measuring_overcrowd-

ing

in_hsg.pdf, 2007.

Bose, M., Olivan, B. and Laferrere, B. Stress and obesity: the role of the hypothalamic-pituitary-adrenal axis in metabolic disease. Curr. Opin. Endocrinol. Diabetes Obes., 2009, 16: 340-346.

Botoseneanu, A. and Liang, J. The effect of stability and change in health behaviors on trajectories of body mass index in older Americans: a 14-year longitudinal study. J. Gerontol. A Biol. Sci. Med. Sci., 2012, 67: 1075-1084.

Brouillette, R. T., Horwood, L., Constantin, E., Brown, K. and Ross, N. A. Childhood sleep apnea and neighborhood disadvantage. J. Pediatr., 2011, 158: 789-795.

Cardoso, M. R., Cousens, S. N., De Goes Siqueira, L. F., Alves, F. M. and D'angelo, L. A. Crowding: risk factor or protective factor for lower respiratory disease in young children? BMC Public Health, 2004, 4: 19.

Chambers, E. C., Schechter, C., Tow, A., Torrens, L., Kohlieber, R. and Calderon, R. Household density and obesity in young black and white adults. Ethn. Dis., 2010, 20: 366-369.

Chami, H. A., Resnick, H. E., Quan, S. F., et al. Association of incident cardiovascular disease with progression of sleep-disordered breathing. Circulation, 2011, 123: 1280-1286.

Denny, F. W. The clinical impact of human respiratory virus infections. Am. J. Respir. Crit. Care Med., 1995, 152: S4-S12.

Desantis, A. S., Diez Roux, A. V., Moore, K., Baron, K. G., Mujahid, M. S. and Nieto, F. J. Associations of neighborhood characteristics with sleep timing and quality: the Multi-Ethnic Study Of Atherosclerosis. Sleep, 2013, 36: 1543-1551.

Evans, G. W., Palsane, M. N., Lepore, S. J. and Martin, J. Residential density and psychological health: the mediating effects of social support. J. Pers. Soc. Psychol., 1989, 57: 994-999.
Fonseca, W., Kirkwood, B. R., Victora, C. G., Fuchs, S. R., Flores, J. A. and Misago, C. Risk factors for childhood pneumonia among the urban poor in Fortaleza, Brazil: a case-control study. Bull. World Health Organ., 1996, 74: 199-208.

Fuller, T. D., Edwards, J. N., Sermsri, S. and Vorakitphokatorn, S. Housing, stress, and physical well-being: evidence from Thailand. Soc. Sci. Med., 1993, 36: 1417-1428.

Gove, W. R., Hughes, M. and Galle, O. R. Overcrowding in the home: an empirical investigation of its possible pathological consequences. Am. Sociol. Rev., 1979, 44: 59-80.

Graham, N. M. The epidemiology of acute respiratory infections in children and adults: a global perspective. Epidemiol. Rev., 1990, 12: 149-178.

Grandner, M. A., Petrov, M. E., Rattanaumpawan, P., Jackson, N., Platt, A. and Patel, N. P. Sleep symptoms, race/ethnicity, and socioeconomic position. J. Clin. Sleep Med., 2013, 9: 05A-05D.

Hale, L. and Do, D. P. Racial differences in self-reports of sleep duration in a population-based study. Sleep, 2007, 30: 10961103.

Hale, L., Hill, T. D., Friedman, E., et al. Perceived neighborhood quality, sleep quality, and health status: evidence from the Survey of the Health of Wisconsin. Soc. Sci. Med., 2013, 79: 16-22.

Imai, K., Keele, L. and Tingley, D. A general approach to causal mediation analysis. Psychol. Methods, 2010a, 15: 309-334.

Imai, K., Keele, L. and Yamamoto, T. Identification, inference and sensitivity analysis for causal mediation effects. Stat. Sci., 2010b, 25: 51-71.

Kapur, V., Blough, D. K., Sandblom, R. E., et al. The medical cost of undiagnosed sleep apnea. Sleep, 1999, 22: 749-755.

Krieger, J. and Higgins, D. L. Housing and health: time again for public health action. Am. J. Public Health, 2002, 92: 758-768.

Leventhal, T. and Brooks-Gunn, J. Moving to opportunity: an experimental study of neighborhood effects on mental health. Am. J. Public Health, 2003, 93: 1576-1582.

Millman, R. P., Carlisle, C. C., Mcgarvey, S. T., Eveloff, S. E. and Levinson, P. D. Body fat distribution and sleep apnea severity in women. Chest, 1995, 107: 362-366.

Mooney, S. J., Richards, C. A. and Rundle, A. G. There goes the neighborhood effect: bias owing to nondifferential measurement error in the construction of neighborhood contextual measures. Epidemiology, 2014, 25: 528-535.

Murtagh, P., Cerqueiro, C., Halac, A., Avila, M., Salomon, H. and Weissenbacher, M. Acute lower respiratory infection in Argentinian children: a 40 month clinical and epidemiological study. Pediatr. Pulmonol., 1993, 16: 1-8.

Ompad, D. C., Galea, S., Caiaffa, W. T. and Vlahov, D. Social determinants of the health of urban populations: methodologic considerations. J. Urban Health, 2007, 84: i42-i53.

Patel, N. P., Grandner, M. A., Xie, D., Branas, C. C. and Gooneratne, $\mathrm{N}$. 'Sleep disparity' in the population: poor sleep quality is strongly associated with poverty and ethnicity. BMC Public Health, 2010, 10: 475.

Peppard, P. E., Young, T., Barnet, J. H., Palta, M., Hagen, E. W. and Hla, K. M. Increased prevalence of sleep-disordered breathing in adults. Am. J. Epidemiol., 2013, 177: 1006-1014.

Peppard, P. E., Young, T., Palta, M., Dempsey, J. and Skatrud, J. Longitudinal study of moderate weight change and sleep-disordered breathing. JAMA, 2000, 284: 3015-3021.

Platt, A. B., Field, S. H., Asch, D. A., et al. Neighborhood of residence is associated with daily adherence to CPAP therapy. Sleep, 2009, 32: 799-806.

Pranathiageswaran, S., Badr, M. S., Severson, R. and Rowley, J. A. The influence of race on the severity of sleep disordered breathing. J. Clin. Sleep Med., 2013, 9: 303-309. 
Redline, S., Tishler, P. V., Schluchter, M., Aylor, J., Clark, K. and Graham, G. Risk factors for sleep-disordered breathing in children. Associations with obesity, race, and respiratory problems. Am. J. Respir. Crit. Care Med., 1999, 159: 1527-1532.

Rohe, W. M. and Han, H. S. Housing and health: time for renewed collaboration. N. C. Med. J., 2012, 73: 374-380.

Ruiter, M. E., Decoster, J., Jacobs, L. and Lichstein, K. L. Sleep disorders in African Americans and Caucasian Americans: a metaanalysis. Behav. Sleep Med., 2010, 8: 246-259.
Spilsbury, J. C., Storfer-Isser, A., Kirchner, H. L., et al. Neighborhood disadvantage as a risk factor for pediatric obstructive sleep apnea. J. Pediatr., 2006, 149: 342-347.

Tingley, D., Yamamoto, T., Hirose, K., Keele, L. and Imai, K. 'Mediation': $R$ package for causal mediation analysis. $R$ package version 4.3.1, 2013.

Young, T., Palta, M., Dempsey, J., Skatrud, J., Weber, S. and Badr, $\mathrm{S}$. The occurrence of sleep-disordered breathing among middleaged adults. N. Engl. J. Med., 1993, 328: 1230-1235. 